\title{
A novel time-varying bullwhip effect metric. An application to promotional sales
}

\author{
Juan R. Trapero ${ }^{\mathrm{a}, *}$, Diego J. Pedregal ${ }^{\mathrm{a}}$ \\ ${ }^{a}$ Universidad de Castilla-La Mancha \\ Departamento de Administracion de Empresas, Ciudad Real 13071, Spain
}

\begin{abstract}
Bullwhip effect is a problem of paramount importance that reduces competitiveness of supply chains around the world. A significant effort is being devoted by both practitioners and academics to understand its causes and to reduce its pernicious consequences. Nevertheless, limited research has been carried out to analyze potential metrics to measure it, that typically are summarized in the coefficient of variation ratio of different echelons demand. This work proposes a new metric based on a time-varying extension of the aforementioned bullwhip effect metric by employing recursive estimation algorithms expressed in the State Space framework to provide at each single time period a real-time bullwhip effect estimate. In order to illustrate the results, a case study based on a serially-linked supply chain of two echelons from the chemical industry is analyzed. Particularly, this metric is employed to analyze the effect of promotional campaigns on the bullwhip effect on a real-time fashion. The results show that, effectively, the bullwhip effect is not constant along time, but interestingly, it is reduced during the promotional periods and it is bigger before and after the promotion takes place.
\end{abstract}

Keywords: Bullwhip effect, metrics, promotions, supply chain management

\section{Introduction}

The bullwhip effect refers to increasing variability of demand from downstream to upstream in the supply chain (Forrester, 1961). This effect pro-

\footnotetext{
${ }^{*}$ Corresponding author.

Email addresses: juanramon.trapero@uclm.es (Juan R. Trapero), diego.pedregal@uclm.es (Diego J. Pedregal)
} 
duces an increase in holding costs, lost sales, low service levels, and a reduced productivity among other effects (see Cannella et al. (2013) and references herein). Despite the fact that the bullwhip effect is considered one of the main problems in Supply Chain Management, the literature about how to measure such an effect has received very limited attention (Fransoo and Wouters, 2000; Cannella et al., 2013).

In general terms, the alternatives to quantify the bullwhip effect can be summarized in two research streams. On the one hand, a theoretical expression may be achieved by assuming: i) a forecasting technique that usually is either an exponential smoothing algorithm (Chen et al., 2000) or autoregressive and moving average models (Duc et al., 2008); and ii) a replenishment policy (commonly an order-up to level stock control). Dejonckheere et al. (2003) also proposed a frequency domain point of view, where engineering tools as the frequency response plot and the periodogram can be employed to determine the bullwhip effect without relying on any assumption regarding the distribution of demand. In addition, they provide different metrics as the Amplitude Ratio; the peak Amplitude Ratio; and the noise bandwidth.

On the other hand, an empirical approach to measure the bullwhip effect may be calculated as the ratio between the demand coefficient of variation and orders coefficient of variation for a determined echelon in the supply chain (Fransoo and Wouters, 2000). This approach does not need to know what are either the forecasting or stock policies employed and it is based solely on measured data. In that sense, let the Bullwhip Ratio $(B W R)$ be defined as:

$$
B W R=\frac{\sigma_{o} / \mu_{o}}{\sigma_{d} / \mu_{d}}
$$

where $\sigma_{o}$ is the standard deviation of the orders signal and $\mu_{o}$ is its mean. Likewise, $\sigma_{d}$ and $\mu_{d}$ are the standard deviation and mean of the market demand signal. Assuming that the orders mean and demand mean are the same, some authors compute the bullwhip effect as the ratio of either standard deviations or variances (Dejonckheere et al., 2003; Trapero et al., 2012). Note that, although in an idealistic situation, empirical and theoretical values of the bullwhip effect should be the same, there have been several studies that show a considerable gap between them, (Zotteri, 2013; Trapero et al., 2014a), where other exogenous factors as the potential incentives to the sales force can play an important role.

In order to bridge the gap between theoretical and empirical bullwhip 
values, a dynamic bullwhip metric capable of estimating the bullwhip effect at each single time period can be helpful to distinguish the causes of such a discrepancy. An important direct application is, for instance, promotional sales. Let's assume that a promotional period takes place and thus, the demand (orders) mean and standard deviation are expected to be timevarying (Blattberg et al., 1995; Trapero et al., 2014b). Expression in (1) is not adequate since the statistics (either standard deviation or mean) are not constant. In other words, when the promotion is active a change of mean and standard deviation can happen, and when the promotion has been exhausted another change may occur. In order to understand how those price variations affect at the bullwhip effect a new empirical time-varying bullwhip metric should be defined. Recall that price variations as well as demand signal processing, rationing gaming, and order batching are the main causes for the bullwhip effect (Lee et al., 1997). Furthermore, if we are able to define a time-varying (local) bullwhip ratio metric, it would facilitate to distinguish which of the potential causes of the bullwhip effect is more significant. It should be noted that this new metric follows the suggestion pointed out by Fransoo and Wouters (2000), where a bullwhip measure should be capable of analyzing which parts of the overall effect are the results of the different causes.

The aim of this paper is twofold. Firstly, a time-varying bullwhip effect metric is provided by means of recursive algorithms as the Kalman Filter and the Fixed Interval Smoothing. Secondly, a case study with promotional sales periods will be assessed in order to illustrate the advantages of adopting a time-varying bullwhip effect metric.

This article is organized as follows: Section 2 introduces the case study and carries out an exploratory data analysis. Section 3 describes the new time-varying bullwhip metric. Section 4 verifies the usefulness of the proposed approach by comparing the results with the traditional metric for data at SKU level subject to promotional campaigns. Finally, main conclusions are drawn in Section 5.

\section{Case study}

The supply chain system consists of a serially linked two-level supply chain. This supply chain comprises a flow of information from the market towards the manufacturer and a reverse one regarding materials. Market sales and shipments from the manufacturer are the measured variables. Note 
that, unless company's fill rate is always $100 \%$, either orders or sales do not reveal the true demand, which is unknown, and they are only estimations (Gilliland, 2010). Data from a manufacturing company specialized in household products have been collected. The data have been sampled weekly from October 2008 to October 2009. This manufacturing company provides products to one of the largest retailers in the UK. The dataset used for this study is composed of three time series: i) manufacturer shipments, which estimates retailers demand; ii) retailer sales, which analogously estimates the market demand ; and iii) a dummy variable that is equal to 1 when a promotion of any kind is active, and 0 otherwise.

The dataset under study comprises 16 Stock Keeping Units (SKU) with 52 observations per SKU. Four different SKU examples are depicted in Fig. 1 , where retailer sales are in a solid line and manufacturer shipments are in a dashed line. Note that the shipments variability tends to be higher than retailers one providing evidence of the BE. The two upper subplots represent two examples without promotions, whereas the two lower subplots in the same figure show two examples with promotional periods highlighted with a grey shadow. It can be observed a sales increase on promotional periods.

Figure 2 shows a histogram with the bullwhip ratio calculated according to (1) for our dataset. In summary, all the SKUs present a bullwhip phenomenon given that the bullwhip ratio is greater than 1 . In fact, most SKUs have a bullwhip ratio between 1.5 and 3, and two SKUs provide a bullwhip ratio greater than 4.5 .

\section{Methodology}

The problem then consists of estimating the time-varying bullwhip effect as the ratio of the shipments coefficient of variation over the sales coefficient of variation, such us:

$$
B W R_{t}=\frac{\sigma_{o t} / \mu_{o t}}{\sigma_{d t} / \mu_{d t}}
$$

where $B W R_{t}$ is the dynamic version of the constant $B W R$ defined previously in (1). The time variation of the bullwhip effect, consequently, may come from different sources. It may vary because the means of shipments $\left(\mu_{o t}\right)$ and/or sales $\left(\mu_{d t}\right)$ are time-varying and/or the standard deviations of shipments $\left(\sigma_{o t}\right)$ and/or sales $\left(\sigma_{d t}\right)$ are time-varying (please beware than all 

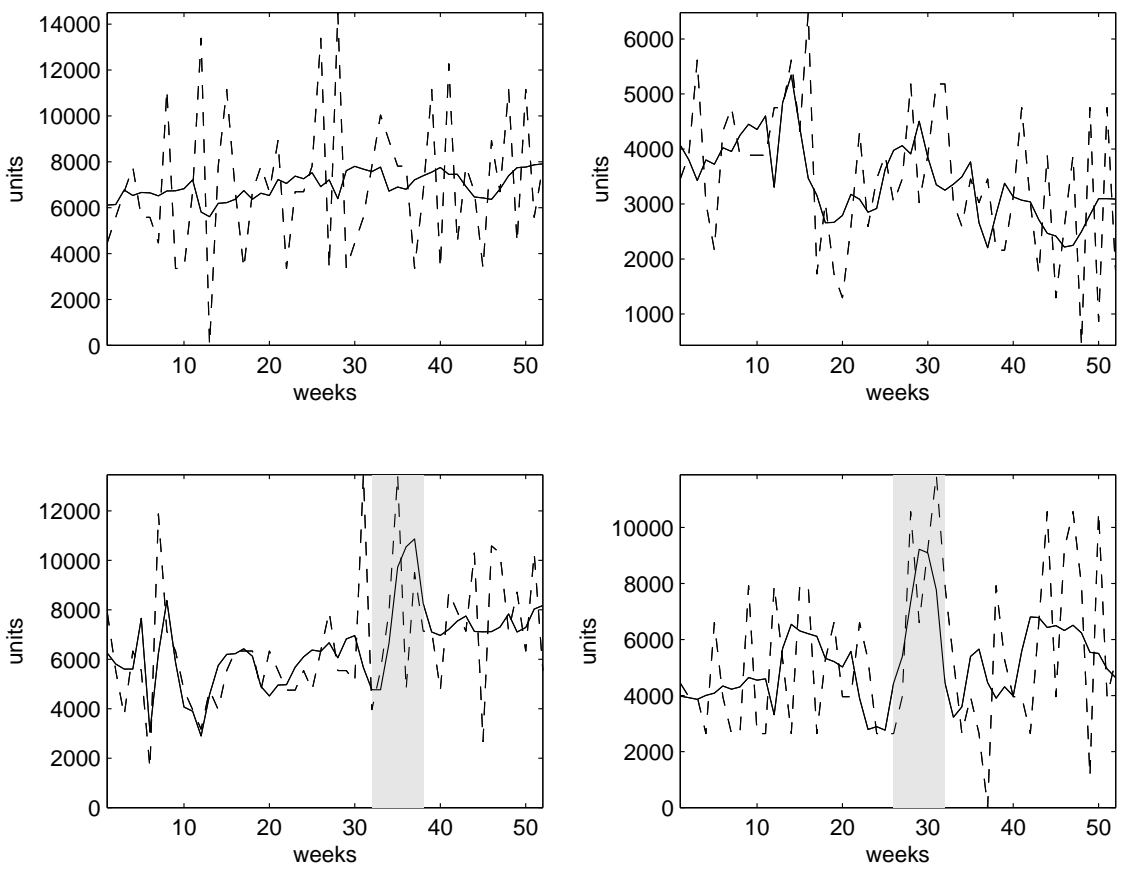

Figure 1: Four SKU examples. Retailer sales are in a solid line and shipments in a dashed line. Promotional weeks are highlighted in a grey area

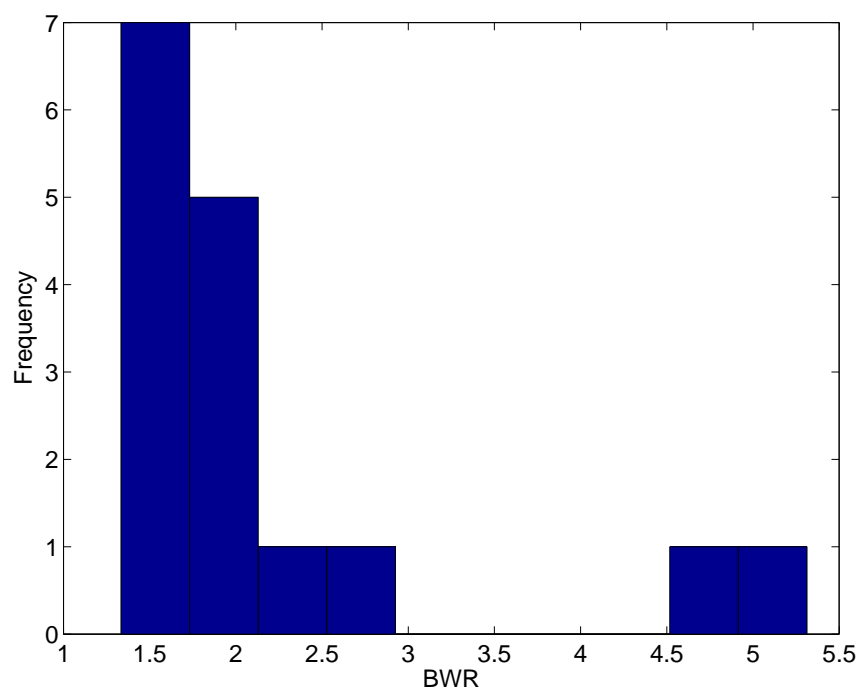

Figure 2: Histogram of the bullwhip ratio. 
"and/or" are necessary in this sentence). Therefore, the problem of estimating a time-varying bullwhip effect relies on estimating time-varying means and standard deviations of shipments and sales.

\subsection{Estimation of the time-varying mean}

One possibility is using a local level model, which smoothed version produces a time-varying mean of a time series based on the whole dataset (Harvey, 1989). The local level model is posed in the State Space framework as follows:

$$
\begin{aligned}
\mu_{t+1} & =\mu_{t}+\epsilon_{t} \\
y_{t} & =\mu_{t}+\nu_{t}
\end{aligned}
$$

where $y_{t}$ is the signal (either shipments or sales), $\mu_{t}$ is the local level, and $\epsilon_{t}$ and $\nu_{t}$ are assumed Gaussian noises with zero mean and a constant variance ratio given by:

$$
q=\frac{\operatorname{Var}\left(\epsilon_{t}\right)}{\operatorname{Var}\left(\nu_{t}\right)}
$$

The first line in equation (3) represents the State Equation and defines the stochastic behavior of the local level, the bigger the variance $\operatorname{Var}\left(\epsilon_{t}\right)$ is, the more rapid the adaptation to changes in mean occurs. The second line shows how the signal is related to the local level, i.e. the data is decomposed into the local mean plus noise. Standard application of the theory of Gaussian linear State Space systems provides the optimal smoothed estimates for the unobserved local level. Estimation of the unknown $q$ may be done by Exact Maximum Likelihood and the Kalman Filter and State Fixed Interval Smoothing would produce the optimal results in this context (a rather comprehensive approach to this kind of modelling is (Pedregal and Young, 2002)). The fact that the ratio $q$ is estimated instead of fixed by some criterion has the advantage that it is the data which says whether the mean is constant (with $q=0$ ) or time-varying $(q>0)$, without any intervention of the researcher. One typical case of the application of this model to SKU data produces the results in Figure 3. In that figure, the time-varying mean is shown in a thicker line, where shipments mean in the upper plot seems slightly decreasing over time, while sales in the lower plot tend to show a steeper decrease rate over time. 

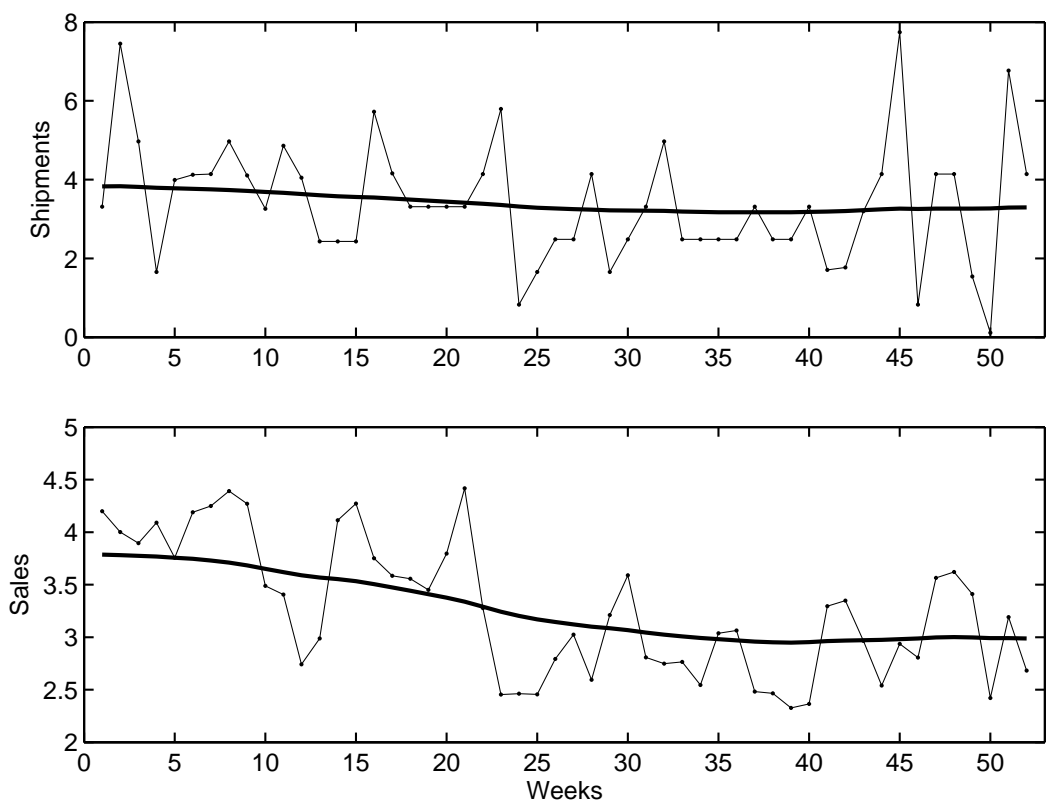

Figure 3: Shipments (upper plot) and sales (lower plot) time-varying mean estimation for a single SKU

\subsection{Estimation of the time-varying standard deviation}

We need to proceed one step further in order to estimate a time-varying standard deviation of the time series. According to Durbin and Koopman (2012), one straightforward way to do it is to smooth the absolute value of the deviations of the data with respect to the time-varying mean with model (3), i.e.:

$$
\begin{aligned}
s_{t} & =\left|y_{t}-\mu_{t}\right| \\
\sigma_{t+1} & =\sigma_{t}+\eta_{t} \\
s_{t} & =\sigma_{t}+\lambda_{t}
\end{aligned}
$$

Analogously, $\eta_{t}$ and $\lambda_{t}$ are assumed Gaussian noises with with zero mean and a constant variance ratio given by:

$$
q^{*}=\frac{\operatorname{Var}\left(\eta_{t}\right)}{\operatorname{Var}\left(\lambda_{t}\right)}
$$



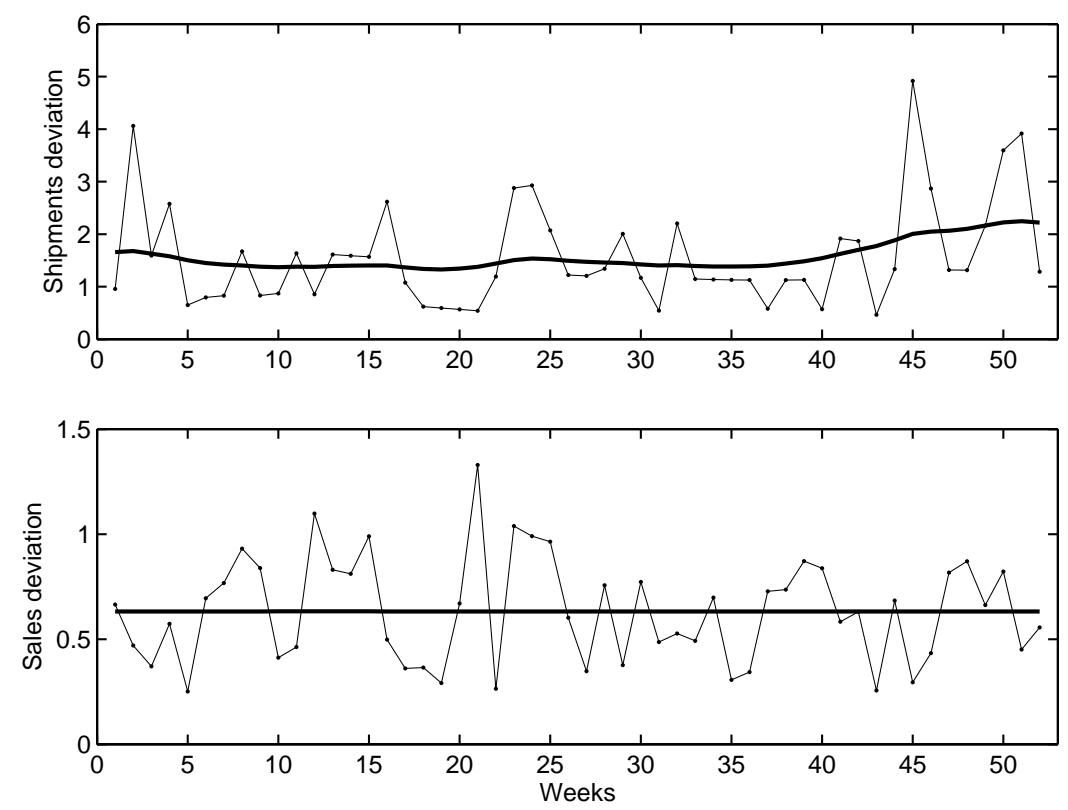

Figure 4: Time-varying standard deviation estimates for the same SKU shown in Figure 3

The time-varying standard deviation estimates for the same SKU in Figure 3 are shown in Figure 4. Again, the estimate according to (5) is represented in a thicker line. Note that, the absolute value of the deviation of shipments and sales according to the first equation in (5) is depicted in a thinner line. The lower plot in that figure shows that sales standard deviation remains approximately constant, while shipments in the upper plot show up some variation.

\subsection{Estimation of the time-varying bullwhip effect metric}

The final step consists of computing the time-varying bullwhip effect in (2) with the previous estimations developed in (3) and (5). The bullwhip effect constant estimation (see (1)) and the time-varying estimation (see (2)) for the SKUs shown in previous figures are depicted in Figure 5. It is clear that the constant estimation provides in general the mean level, but does not take into account the possible variations in time. In the particular case shown in Figure 5, there is a clear trend to an increase in the bullwhip effect, mainly motivated by the increase of shipments standard deviation. 


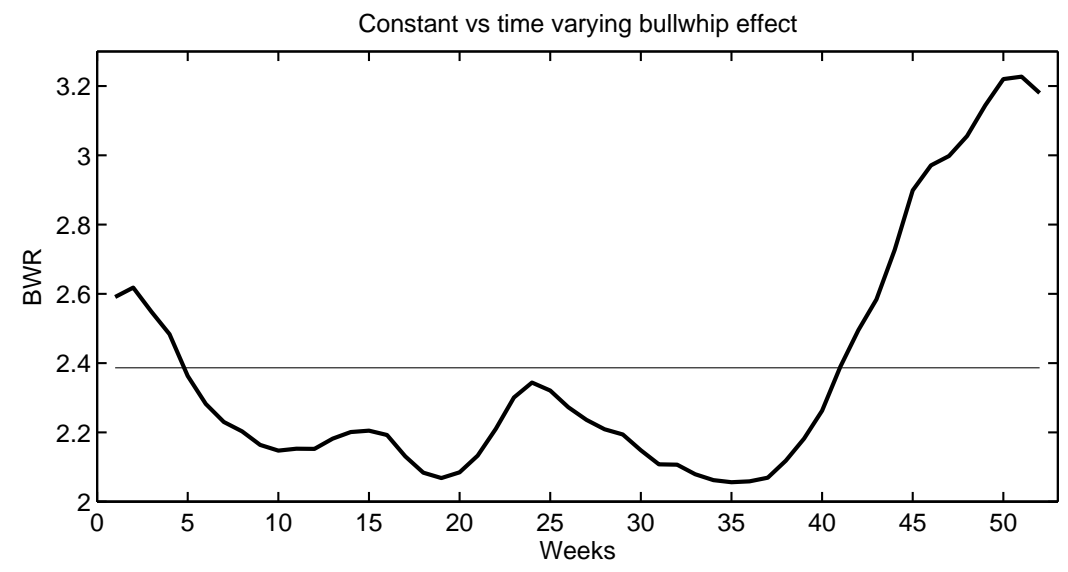

Figure 5: Constant bullwhip effect estimate (thin line) vs. time-varying estimate (thick line) for SKU shown in Figures 3 and 4.

Figure 6 presents four additional cases of time-varying estimation of different nature, one of them wandering about the mean with important variations, some of them trending downwards, another one trending upwards.

\section{Experimental results on promotional sales}

Price variation is considered a significant cause of the bullwhip effect (Lee et al., 1997). Nonetheless, there is a lack of literature that quantifies such an assumption. In fact, Hamister and Suresh (2008) suggest that with a serially correlated demand process $(\mathrm{AR}(1))$, the use of dynamic pricing may actually reduce the bullwhip effect. This conclusion relaxes the initial assumption that stable pricing always reduce the bullwhip effect. In this sense, the traditional bullwhip effect metric shown in (1) is not adequate to estimate the transitory effect of the promotions on the bullwhip effect. In order to shed some light on this matter, the dynamic bullwhip effect metric in (2) is proposed.

The easiest way is to apply the approach developed in the previous section to the data as it is, i.e. assuming that the promotional dates are unknown. But a second option is to consider that such promotions are known in advance. Note that such an assumption is valid in this case study, since the duration and terms of promotions are most often part of an accepted promotional schedule. The second choice may be modeled by adding a step dummy variable to the model above for the weeks where the promotion is active, such 

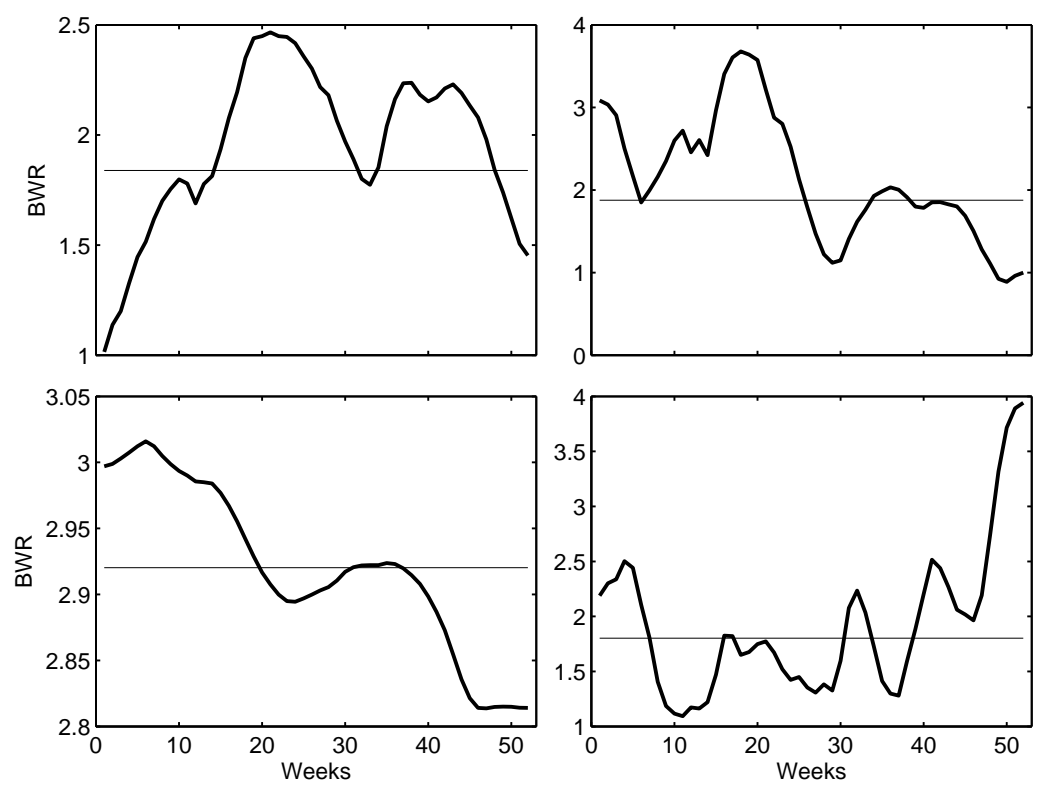

Figure 6: Four SKU examples that support the time-varying behaviour of the bullwhip effect.

that the state is allowed to jump suddenly towards the mean levels at the beginning and at the end of each promotion. System (3) then changes to (7), with one additional parameter $b$ that affects the step variable $u_{t}$.

$$
\begin{aligned}
\mu_{t+1} & =\mu_{t}+b u_{t}+\epsilon_{t} \\
y_{t} & =\mu_{t}+\nu_{t}
\end{aligned}
$$

One example of the estimation of system (3) and (7) on actual data subject to promotional periods is shown in Figure 7. The estimation ignoring the promotion using model (3), which it is represented in a dashed line, produces less smooth estimates because the higher volatility induced by the promotion results in a greater $q$. In contrast, estimation provided by model (7) (plotted in a thick solid line) allows to jump suddenly at the time of promotion, removing part of such variation. What is rather interesting is that, independently of the estimation, the bullwhip effect if smaller during the promotion than in the rest of the sample. As a matter of fact, the effect seems to amplify before and/or after the promotion.

The results repeat systematically in the rest of cases at hand. Four of them are shown in Figure 8. In all cases except the last one, the bullwhip 


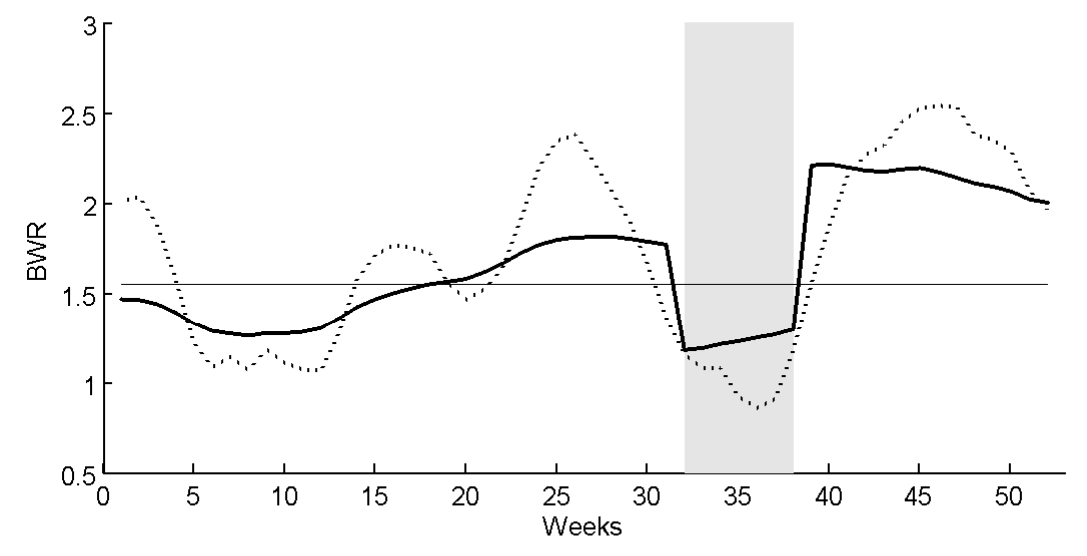

Figure 7: Estimation of time-varying bullwhip effect in the presence of a promotion (shaded area). Model (3) and (7) are represented in a thick dashed and solid line, respectively.The constant bullwhip estimate is plotted in a thin line

effect is smaller, regardless of the estimation with model (3) or (7) and the effect is higher generally after the promotion, and sometimes before. The latter case (bottom right panel) shows a case with three promotions separated by a few weeks. The previous conclusions apply to the first promotion, but not to the subsequent ones.

\section{Conclusions}

A new bullwhip effect metric based on recursive estimation algorithms is proposed. Essentially, the traditional constant bullwhip effect metric is reviewed as a particular case of a more general algorithm capable of handling time-varying estimations. This novel formulation is important since it makes possible to get a deeper insight about the potential causes of the bullwhip effect changes.

A direct application of the proposed metric on promotional sales at SKU level is also investigated. Data from a two echelon supply chain is utilized in this study. Given that SKUs subject to promotional activities are expected to have time-varying sales and shipments, the dynamic bullwhip effect metric is well-suited to analyze the impact of promotional campaigns on the bullwhip effect. The estimation results show that during the promotional periods the bullwhip effect is reduced and it is before and after then, when the bullwhip 

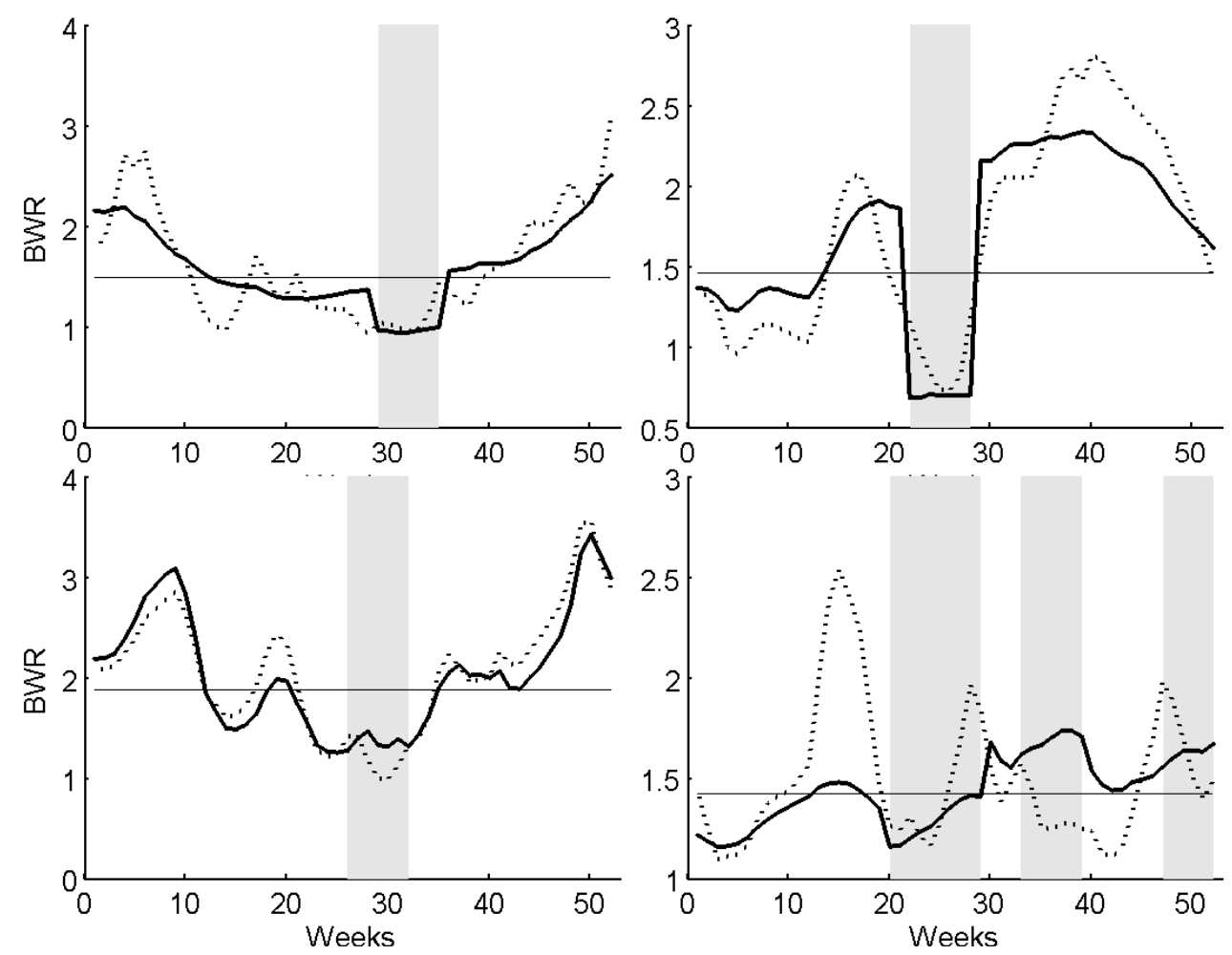

Figure 8: Estimation of time-varying bullwhip effect in the presence of promotional activities (shaded areas) for four different SKUS. Model (3) and (7) are represented in a thick dashed and solid line, respectively. The constant bullwhip estimate is plotted in a thin line 
effect is increased. Note that this conclusion expand our knowledge on the effect of marketing campaigns on the bullwhip effect.

Further research should revisit the influence of bullwhip effect causes as order batching, forecasting methods or stock policies by using the proposed dynamic metric, as well as, more case studies from different industries are required to make these results more solid.

Other possible extension of this work is to apply it to the bullwhip measurement system proposed in Cannella et al. (2013) to make it dynamic. For instance, they propose, among other Key Performance Indicators (KPI), an Inventory Variance Ratio as the coefficient of the inventory variance and the customer demand variance. This KPI is based on the assumption of both constant variance and mean and thus, the time-varying methodology here proposed may be more adequate to make those KPI dynamics and thus, to provide a more valuable information.

\section{Acknowledgment}

This work was supported by the European Regional Development Fund and Spanish Government (MINECO/FEDER, UE) under the project with reference DPI2015-64133-R.

\section{References}

Blattberg, R. C., Briesch, R., Fox, E. J., 1995. How promotions work. Marketing Science 14 (3), G122-G132.

Cannella, S., Barbosa-Póvoa, A. P., Framinan, J. M., Relvas, S., 2013. Metrics for bullwhip effect analysis. Journal of the Operational Research Society $64,1-16$.

Chen, F., Ryan, J. K., Simchi-Levi, D., 2000. The impact of exponential smoothing forecasts on the bullwhip effect. Naval Research Logistics (NRL) 47 (4), 269-286.

Dejonckheere, J., Disney, S. M., Lambrecht, M. R., Towill, D. R., 2003. Measuring and avoiding the bullwhip effect: A control theoretic approach. European Journal of Operational Research 147, 567-590. 
Duc, T. T. H., Luong, H. T., Kim, Y.-D., 2008. A measure of bullwhip effect in supply chains with a mixed autoregressive-moving average demand process. European Journal of Operational Research 187 (1), 243 - 256.

Durbin, J., Koopman, S., 2012. Time Series Analysis by State Space Methods: Second Edition. Oxford Statistical Science Series. OUP Oxford.

Forrester, J. W., 1961. Industrial dynamics. Vol. 2. MIT Press: Cambridge, MA.

Fransoo, J. C., Wouters, M. J. F., 2000. Measuring the bullwhip effect in the supply chain. Supply Chain Management: An International Journal 5 (2), 78-89.

Gilliland, M., 2010. Defining "demand" for demand forecasting. Foresight: The International Journal of Applied Forecasting (18), 4-8.

Hamister, J. W., Suresh, N. C., 2008. The impact of pricing policy on sales variability in a supermarket retail context. International Journal of Production Economics 111 (2), 441 - 455, special Section on Sustainable Supply Chain.

Harvey, A., 1989. Forecasting Structural Time Series Models and the Kalman Filter. Cambridge University Press.

Lee, H. L., Padmanabhan, V., Whang, S., 1997. The bullwhip effect in supply chains. Sloan Management Review Spring, 93-102.

Pedregal, D. J., Young, P. C., 2002. Statistical approaches to modelling and forecasting time series. Companion to Economic Forecasting Blackwell Publishers, Ch. 4, pp. 69-104.

Trapero, J. R., Garcia, F. P., Kourentzes, N., 2014a. Impact of demand nature on the bullwhip effect. bridging the gap between theoretical and empirical research. In: Xu, J., Fry, J. A., Lev, B., Hajiyev, A. (Eds.), Proceedings of the Seventh International Conference on Management Science and Engineering Management. Vol. 242 of Lecture Notes in Electrical Engineering. Springer Berlin Heidelberg, pp. 1127-1137.

Trapero, J. R., Kourentzes, N., Fildes, R., 2012. Impact of information exchange on supplier forecasting performance. Omega 40 (6), 738 - 747, special Issue on Forecasting in Management Science. 
Trapero, J. R., Kourentzes, N., Fildes, R., 2014b. Identification of sales forecasting models. Journal of the Operational Research Society In press, - .

Zotteri, G., 2013. An empirical investigation on causes and effects of the bullwhip-effect: Evidence from the personal care sector. International Journal of Production Economics 143 (2), 489 - 498, focusing on Inventories: Research and Applications. 